\title{
SOME ADVANCES IN PERINATAL CARE ${ }^{\dagger}$
}

\author{
E. J. COETZEE* M.B. Ch.B., M.R.C.O.G.
}

One of the biggest advances in obstetrics has been the use of ultrasound for imaging and measurement of the fetus from the 6th week after menstruation until delivery.

Ultrasound is sound above the audible range. We can hear sound when the frequency is between 16-20000 cycles per second (Herz, Hz). For diagnostic ultrasound work in obstetrics the frequency is $2-3$ million cycles per second (megahertz, MHz).

Before we can accept this new modality we must, however, be sure of its safety. Many workers have confirmed that intensities used in diagnostic work are safe as far as known criteria (e.g. chromosomal breaks) are concerned. In addition the beam of ultrasound is pulsed. A pulse of energy lasts approximately 1 microsecond and such pulses are only repeated $600-1000$ limes per second. It can, therefore, be seen that during the vast majority of time there is no energy output from the machine. It would seem that unlike X-rays, ultrasound can be used repeatedly in pregnancy.

\section{FETAL HEAD MEASUREMENT}

One of the first fetal parts which was clearly visualised even by the first machines used was the fetal head. Because of this the fetal head could be measured. Professor S. Campbell described the standard technique which should be used when measuring the biparietal diameter (the distance between the parietal eminences). There were two important developments from this work. An accurate biparietal diameter could be equated to a gestational age, especially if the measurement was done before 28 weeks. In the second place, the growth of the fetus could be ascertained by repeated measurements of the biparietal diameter. Using this technique important parameters of placental function and fetal well-being could be ascertained. Today, with more sophisticated equipment, the fetal head circumference at the level of the third ventricle and the fetal abdominal circumference at the level of the umbilical vein can be measured, adding important information concerning fetal growth.

\section{DETECTION OF ABNORMALITY}

With sophisticated machinery and the accidental discovery that a full bladder allowed excellent visualisation of the pelvic organs, it became possible to follow the growth of the pregnancy sac and the fetus from the 6 th week of pregnancy. The fetal heart could also be seen from at least the 8 th week of pregnancy and recorded. Because of this advance, the whole management of threatened abortion has been changed and clinicians and patients no longer have to wait in suspense concerning the viability of the pregnancy. Conditions such as a blighted ovum, missed abortion and trophoblastic disease can be diagnosed accurately and quickly.

Another application ' of diagnostic ultrasound is the accurate localisation of the placenta in cases of ante-

*ecturer, Department of Gynaecology and Obstetrics,
University of Cape Town and Senior Specialist,
Groote Schuur Hospital. $\uparrow$ Paper read at Congress preceding 13th National Council Meeting 23-27 April 1979. partum haemorrhage. If the placenta is not lying near or over the cervical os (placenta praevia), in many instances the patient can be allowed to go home instead of spending the last weeks of her pregnancy in hospital.

The diagnosis of intra-uterine demise of the fetus is also possible, as is the detection of certain abnormalities of the fetus such as hydrocephaly, anencephaly, polycystic kidneys, large cysts in the fetal abdomen, and others.

\section{GREY SCALE AND REAL TIME}

The newest developments in ultrasound are grey scale pictures and real time scanners. In the former the size of the reflected ultrasound, and therefore of the electrical impulse generated at the crystal, is related to a certain shade of grey which gives far more detail than the previous simple black and white picture. In real time scanning, new pictures are created all the time and as with a cine camera movement of the fetus can be seen. The fetus can now be seen to hiccup, breathe, move his limbs and do total body movements.

\section{PRENATAL DIAGNOSIS}

A second major development is the use of prenatal diagnosis and the detection of certain fetal abnormalities. Chromosomal abnormalities, as in Down's syndrome, neural tube defects and a host of enzyme deficiency diseases can now be detected by performing amniocentesis between the 16th and 20th weeks. Ultrasound has played a major part in making amniocentesis safer. Before inserting the needle through the abdominal and uterine walls into the amniotic fluid, the position of the fetus and the placenta is determined by ultrasound and the appropriate spot is marked on the patient's abdomen where there will be reasonably safe and direct access to amniotic fluid. With the use of ultrasound, the percentage of failures is reduced, the number of needle insertions is reduced, and clean liquor, uncontaminated with blood, is usually obtained. It is likely that the risk of abortion is also reduced.

The amniotic fluid is then sent to the human genetics laboratory where the alpha-fetoprotein is measured for detection of neural tube defects, such as spina bifida and anencephaly. There are also viable fetal cells in this amniotic fluid which, by careful techniques, can be induced to multiply; chromosomal studies can be done on the cultured cells. The best known and commonest chromosomal abnormality is Down's syndrome, in which there are 3 chromosomes in the 21 chromosome set (trisomy-21). Other rarer chromosomal abnormalities can also be detected. The incidence of Down's syndrome increases with maternal age, and at the maternal age of 40 approximately 1 in 50 preg. nancies could result in Down's syndrome. Before offering prenatal diagnosis one must however consider that there is a $1 \%$ risk of the patient's aborting because of the procedure. The chance of detecting an abnormal fetus should therefore be at least $1 \%$. Under South African law, a pregnancy with an abnormal fetus can be terminated, and the tragedy of an abnormal child thus averted.

In the late midtrimester and early third trimester, amniocentesis is important in the management of complicated rhesus affected pregnancies. The severity 
of the haemolysis in the fetus can be ascertained by measuring bilirubin-like pigments in the amniotic fluid.

But the most important indication for amniocentesis is the prevention of respiratory disease in the newborn. Liquor is usually taken by suprapubic puncture, but sometimes under ultrasound guidance; $1 \mathrm{ml}$ liquor is mixed with $1 \mathrm{ml}$ alcohol and shaken up. The amount of stable bubble formation tells us whether the fetus has surfactant or not, which will protect it from respiratory distress syndrome.

\section{FETAL MONITORING}

A further invasion into the privacy of the fetal world has taken place in the labour ward. To make labour safer for mother and fetus, monitors have been developed which can monitor the mother's contractions and record the fetal heart rate continuously. The nor- mal fetal heart rate is between 120 and 160 beats ner minute and the baseline should be changing all the time. Normally there is no relationship between the fetal heart and contractions. The following changes are associated with fetal distress. A fetal heart rate con. tinuously above 160 (tachycardia), or a heart rate continuously below 120 (bradycardia), suggests distress. Absence of baseline variability is an ominous sign. Often, especially near delivery, the fetal heart rate will decelerate with the contraction, but rccover immediately. This is due to compression of the head. However, when the deceleration continues after the contraction has ended, this is a particularly ominous sign of fetal distress.

When the obstetrician is worried about the fetal heart rate pattern, he will take a sample of blood from the fetal scalp and estimate its $\mathrm{pH}$ value. If the pH is below 7,2 this is almost always an absolute sign of a fetal problem; delivery should be at once expedited, usually by caesarean section.

\section{REPORT ON CONGRESS PROCEEDINGS}

\section{INTRODUCTORY COURSE IN NEURODEVELOPMENTAL THERAPY}

37 members attended this course. The lectures and demonstrations were given by members of the South African Neurodevelopmental Therapy Association. The first session discussed the properties of spasticity and the problems which spasticity poses to the physiotherapist. Current methods of controlling spasticity were analysed and the underlying philosophy of neurodevelopmental therapy was presented. The following session formulated the role played by the postural reflex mechanisms in determining normal motor development. The demonstration which followed showed how these postural reflex mechanisms can be utilized to obtain specific movement patterns in preparation for future skills as well as functional sequences of movement. The second half of the course was devoted to analysing the functional problems encountered with sitting standing and walking in different types of spastic patients. This section included an outline of how these problems develop as well as guidelines for treatment.

The workshop on upper extremity function was not held as planned owing to lack of support from Society members. Instead, an informal workshop was thrown open to occupational, speech and physical therapists and also teachers - and over 30 people attended two separate sessions, the first devoted to analysing upper extremity functions in the normal as well as in spastic and athetoid children and the second to discussing how to establish these functions in spastic children and in athetoid children.

S. Irwin-Carruthers

\section{WORKSHOP ON CLINICAL SUPERVISION}

The first session of the workshop which was chaired by Mrs. A. Mathias, Chief Physiotherapist of the Johannesburg Hospital complex, commenced with group formulation of aims and objectives of clinical supervision. The most important aspect that emerged from these groups was realisation that the clinical situation was the prime area for growth and development of the student.

By providing the right climate in the clinical situation, the student will be stimulated to think independently, to use her initiative and want to probe further and be responsible for her own learning. By teaching through demonstration, the supervisor will encourage acceptable standards of interpersonal relationships and professional values as well as reinforce in practice that which is taught in the classroom.

In two very competent talks given by students from the Universities of Cape Town and Stellenbosch, the need for better communication between student and supervisor was stressed. Claire Johnson urged far greater involvement of the supervisor in the clinical situation and felt that the student would only be able to learn, if she knew what was done correctly, what was unacceptable and how this could be changed.

Carin Hugo urged greater commitment on the part of the supervisor to provide assistance in all aspects of patient handling by demonstration and discussion, especially when the student is at the beginning of her clinical training. The student has the right to know what is good or bad in her patient management and must be given the chance to correct mistakes as well as specify her own problems. Evaluation must be objective and thorough and not be just a mark. Sho pleaded for supervisors to be enthusiastic about theif profession in order to stimulate the students.

In attempting to provide a meaningful learning experience in the clinical field, certain objectives for clinical supervisors were suggested by J. C. Beenhakker. These were teaching by demonstration in all areas of clinical competencies, co-ordinating class teaching and clinical practice, setting goals for achievement in that particular department, developing skills of problemsolving and providing constant and constructive feedback to the student. This feedback to the student is to point out where her strengths and weaknesses lie and whether the student has fulfilled the stated goals. The student should also be encouraged to assess the supervisor in order to bring about desirable change. The ideal supervisor should be competent in her own field. be available at all times, be able to communicate with the student and be sensitive to her needs.

In the second session under the Chairmanship of Miss M. J. Runnalls, Head of the Department of Physiotherapy of the University of Stellenbosch and Tygerberg Hospital, the emphasis was on the methods by which clinical supervision should be carried out. Although P. Bowerbank, Chief Physiotherapist at Kalafong Hospital, presented her talk in the form of a 